Research Article

\title{
Spectral Efficient Asymmetrically Clipped Hybrid FBMC for Visible Light Communication
}

\author{
Sanjeev Kumar (i) and Preeti Singh \\ Department. of ECE, UIET, Panjab University, Chandigarh, India \\ Correspondence should be addressed to Preeti Singh; preeti_singh@pu.ac.in
}

Received 10 September 2020; Revised 3 January 2021; Accepted 9 January 2021; Published 23 January 2021

Academic Editor: Sulaiman W. Harun

Copyright (c) 2021 Sanjeev Kumar and Preeti Singh. This is an open access article distributed under the Creative Commons Attribution License, which permits unrestricted use, distribution, and reproduction in any medium, provided the original work is properly cited.

\begin{abstract}
Filter bank multicarrier (FBMC) modulation has shown sufficient potential for wireless communication. A hybrid optical FBMC technique is proposed to improve the spectral efficiency of a visible light communication (VLC) system. In this technique, a hybrid asymmetrically clipped optical offset quadrature amplitude modulation FBMC (HACO-OQAM-FBMC) modulation technique is used. Asymmetrically clipped optical FBMC (ACO-FBMC) is used for odd subcarriers, and pulse amplitude modulation-discrete multitone (PAM-DMT) is used for the even subcarriers. The proposed hybrid scheme uses an intensity modulation/direct detection (IM/DD) channel. It is shown that there is no interference on odd subcarriers using the proposed method and receiver demodulation is similar to that of ACO-FBMC receiver. However, clipping noise of ACO-FBMC falls on PAM-DMT subcarriers, which can be cancelled at receiver processing after estimation. The analytical performance of the proposed technique is compared using parameters, namely, bit error rate (BER), spectral efficiency, computational complexity, and peak to average power ratio (PAPR). It is found that HACO-OQAM-FBMC is more spectral efficient than ACO-FBMC and other OFDM-based techniques.
\end{abstract}

\section{Introduction}

Orthogonal frequency division multiplexing (OFDM) is used extensively in wired and wireless communication systems. Despite its popularity, it possesses some limitations also such as high out-of-band distortion and long cyclic prefix $(\mathrm{CP})$. In previous years, alternative multicarrier modulation techniques have been studied to address OFDM issues. The filter bank multicarrier (FBMC) modulation technique is considered one of the most potential waveforms for next-generation wireless communications due to its robustness against intersymbol interference (ISI) [1].

Visible light communication (VLC) has drawn great attention of researchers due to its unlicensed spectrum, no health hazards, and no electromagnetic interference [2]. In radio frequency (RF) communications, FBMC has been considered as a strong competitor of OFDM $[3,4]$. But, for the VLC system, which uses an intensity modulation/direct detection (IM/DD) channel, the signal should be real and unipolar [5-7]. Moreover, the average power of RF communication is the mean square value of the signal, but the average power of VLC is the mean value of the signal. A lower average power is usually preferred for RF communication, but VLC has a predefined average intensity according to the dimming target, which is not an objective function but a constraint $[8,9]$. So, FBMC techniques as implemented in RF communications straightforward cannot be used in the VLC system.

The modulation techniques mostly used in the VLC system are asymmetrical clipped optical OFDM (ACOOFDM) and DC biased optical OFDM (DCO-OFDM). Spectral efficiency of ACO-OFDM is low because only odd subcarriers are used and even subcarriers are neglected to meet the requirement of the VLC system [10]. To improve the spectral efficiency of the VLC system mostly proposed techniques in literature are based on OFDM. Asymmetrically clipped DC biased OFDM (ADO-OFDM) to improve the spectral efficiency of ACO-OFDM is proposed in [11]. But, it uses DC bias for even subcarriers which is a power inefficient method. In [12], the author proposed enhanced hybrid asymmetrically clipped OFDM (EHACO-OFDM) which increases spectral efficiency but has more complexity. 
Till date, there is no work reported in the literature which discusses the assessment of the hybrid FBMC modulation technique for VLC based on the IM/DD channel.

In this paper, the authors proposed hybrid asymmetrically clipped optical offset quadrature amplitude modulation FBMC (HACO-OQAM-FBMC) in which ACO-FBMC is used for the odd subcarriers and PAM-DMT is used for the even subcarriers. The clipping noise of ACO-FBMC falls only on even subcarriers. The receiver of HACO-OQAMFBMC consists of four signals: the wanted ACO-FBMC signal, clipping noise from ACO-FBMC, PAM-DMT signal, and noise added in the channel. The clipping noise of ACOFBMC can be accurately separated from PAM-DMT after estimation before demodulation.

The authors' contributions can be summarized as follows:

(1) The system model is proposed for the HACOOQAM-FBMC modulation technique

(2) Scaling techniques are used for the proposed FBMCbased model for the VLC system

(3) The performance analysis is carried out using spectral efficiency, bit error rate (BER), computational complexity, and peak to average power ratio (PAPR) for the FBMC-based VLC system

(4) Non-DC bias methods are used for the proposed model of the VLC system

(5) The performance of ACO-OFDM, DCO-OFDM, ADO-OFDM, EHACO-OFDM, ACO-FBMC, DCOFBMC, and HACO-OQAM-FBMC modulation techniques has been compared

The content of this paper is systematized as follows. Section 2 gives the system model for the HACO-OQAMFBMC modulation technique. Performance analysis of the proposed system models is performed in Section 3 using parameters, probability density function, bit error probability, peak to average power ratio, computational complexity, spectral efficiency, and power spectral density. The conclusion is presented in Section 4 .

\section{Proposed Modulation Techniques System Model}

The system model consists of a transmitter and receiver functional diagram for the VLC system. Figure 1 shows the functional diagram of the transmitter and receiver section of the HACO-OQAM-FBMC modulation technique.

In the transmitter section of HACO-OQAM-FBMC, the offset quadrature amplitude modulation (OQAM) is used for signal mapping. Hermitian symmetry is used to fit the signal into VLC system requirements. Odd subcarriers are modulated using an ACO-FBMC modulator, and a PAMDMT modulator is used for even subcarriers. An optical modulator is employed to convert an electrical signal to optical signal. In the receiver section of HACO-OQAMFBMC, an optical demodulator is used to convert an optical signal back to an electrical signal. For extraction of even and odd subcarriers, ACO-FBMC and PAM-DMT demodulators are used. The equalizer is used to remove the distortion, and finally, the signal is extracted using a decoder. The signal analysis of the HACO-OQAM-FBMC transmitter and receiver is given in the next section.

\subsection{Signal Analysis of the HACO-OQAM-FBMC Transmitter.}

The data subcarriers are given by $Z=\left[Z_{0}, Z_{1}, Z_{2}, \ldots, Z_{N-1}\right]$. The ACO-FBMC consists of odd subcarriers $Z_{\text {odd }}$, and PAMDMT consists of even subcarriers $Z_{\text {even }}$. The even and odd subcarriers are sent to the PAM-DMT and ACO-FBMC modulators which consist of IFFT and clipping of the signal. The transmitted ACO-FBMC signal is represented by

$$
z_{\mathrm{ACO}-\mathrm{FBMC}}=z_{\mathrm{odd}}+n_{\mathrm{afcn}} .
$$
by

Similarly, the transmitted signal of PAM-DMT is given

$$
z_{\mathrm{PAM}-\mathrm{DMT}}=z_{\text {even }}
$$

where $n_{\mathrm{afcn}}$ and $n_{\mathrm{dfcn}}$ are the clipping noise of ACO-FBMC and PAM-DMT [13]. Using equations (1) and (2), the transmitted signal of HACO-OQAM-FBMC is given by

$$
\begin{aligned}
& z(t)=z_{\text {ACO-FBMC }}+z_{\text {PAM-DMT }}, \\
& Z(t)=z_{\text {odd }}+n_{\text {afcn }}+z_{\text {even }} .
\end{aligned}
$$

Upper-case letters are used to represent frequency-domain signals, and lower-case letters are used to represent time-domain signals.

2.1.1. ACO-FBMC Modulator. The OQAM modulator output shown in Figure 1 consists of a real and imaginary signal. Upsampling is performed before prototype filter to increase the sampling rate. A PHYDYAS protype filter is used in the proposed method. After IFFT execution, clipping is required to fulfil optical modulator constraints. The block diagram of the ACO-FBMC modulator is shown in Figure 2.

The PHYDYAS prototype filter [14] is given by

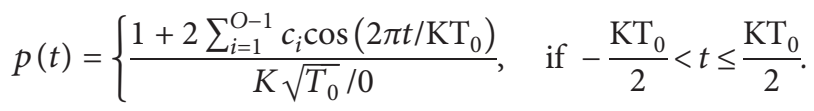

The coefficients $c_{i}$ depend on the overlapping factor $K$, and the procedure for calculation is given in [15]. For example, when the value of the overlapping factor $K=4$, the coefficients are given by

$$
\begin{aligned}
c_{1} & =1.412692577, \\
c_{2} & =1.41421356237 / 2, \\
c & =0.23514695 .
\end{aligned}
$$

Orthogonal: $T=T_{0} ; F=2 / T_{0} \longrightarrow \mathrm{TF}=2$. Localization: $\sigma_{t}=0.2015 T_{0} ; \sigma_{f}=0.403 T_{0}^{-1}$. Joint Localization: $\sigma_{t} \sigma_{f}=1.13 \times(1 / 4 \pi)$.

2.2. Signal Analysis of the HACO-OQAM-FBMC Receiver. The received signal for HACO-OQAM-FBMC is given by 


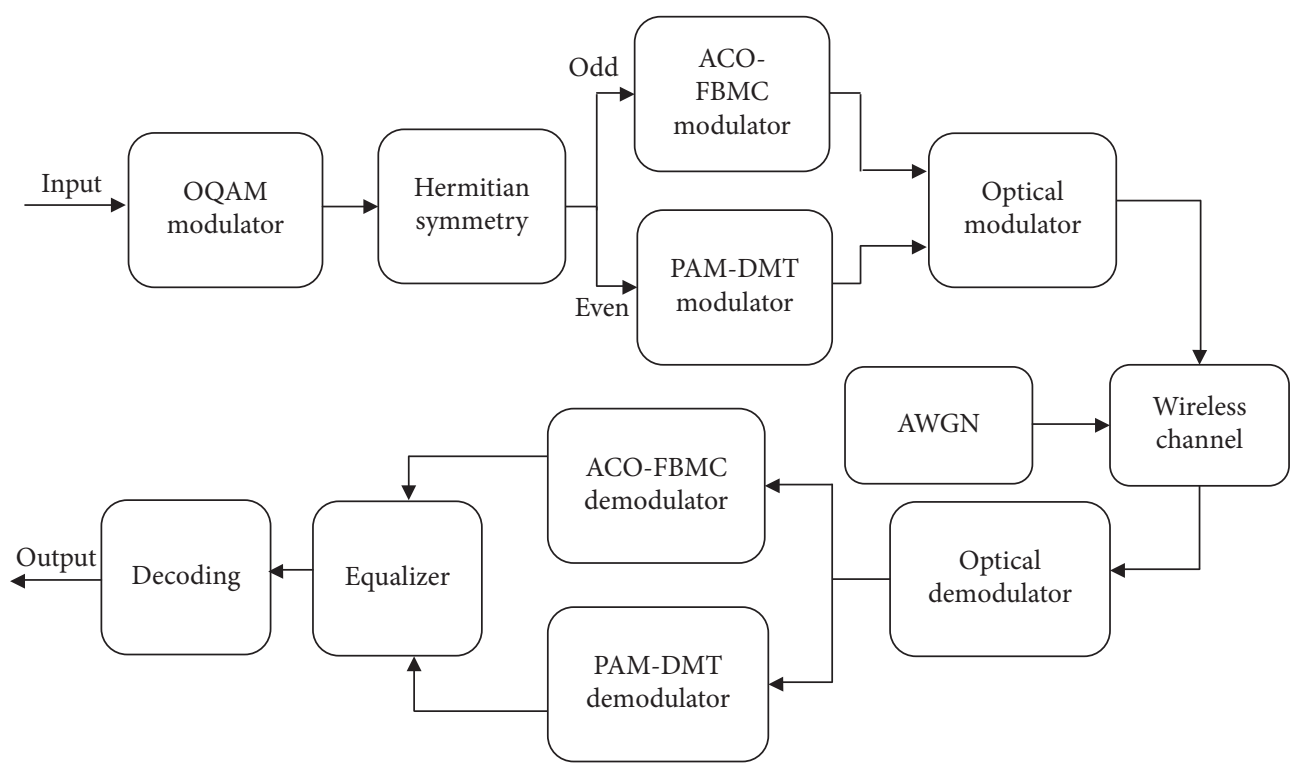

Figure 1: Block diagram of HACO-OQAM-FBMC.

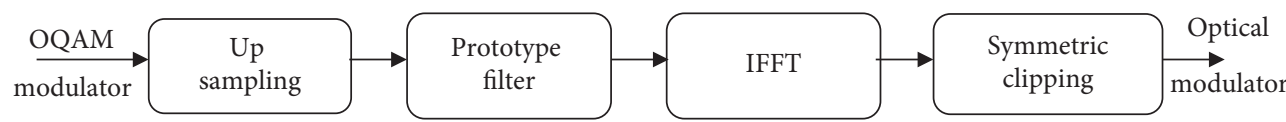

Figure 2: Block diagram of the ACO-FBMC modulator.

$$
y(t)=z_{\mathrm{odd}}+n_{\mathrm{afcn}}+z_{\mathrm{even}}+n_{d_{\mathrm{awgn}}}+n_{a_{\mathrm{awgn}}}
$$

where $n_{d_{\text {_awgn }}}$ and $n_{a_{\text {_awgn }}}$ are the additive white Gaussian noise (AWGN) of the even and odd subcarriers. A flat channel is assumed for one receiver. After applying FFT to equation (6), the signal is given by

$$
Y(t)=Z_{\text {odd }}+N_{\text {afcn }}+Z_{\text {even }}+N_{d_{\text {awgn }}}+N_{a_{\text {awgn }}} .
$$

The clipping noise and AWGN noise are removed using ACO-FBMC and PAM-DMT demodulators. The decoded signal is given by

$$
Y(t)=z_{\text {odd }}+z_{\text {even }}
$$

2.2.1. ACO-FBMC and PAM-DMT Signal Detection. The optical demodulator output, as shown in Figure 1, consist of ACO-FBMC, PAM-DMT, AWGN noise, and clipping noise. The received signal from optical demodulator is converted into frequency domain using FFT. After that, the ACO-FBMC signal is detected. To recover the PAM-DMT signal, we have to use the clipping noise estimation technique, as shown in Figure 3. In this technique, for proper detection and scaling, odd subcarriers are multiplied by 2 because power of the M-QAM symbols reduced by half due to clipping operation $[16,17]$. Subtraction of estimated clipping noise which falls on PAM-DMT (even) subcarriers from received ACO-FBMC symbols will give PAM-DMT symbols.

\section{Performance Analysis of HACO-OQAM- FBMC}

The performance analysis is carried out using probability density function, bit error rate, spectral efficiency, computational complexity, peak to average power ratio, and power spectral density.

3.1. Probability Density Function (PDF) of HACO-OQAM$F B M C$. The PDF of HACO-OQAM-FBMC can be calculated by convolving the PDF of ACO-FBMC and PDF of PAM-DMT. The PDF of ACO-FBMC is given by $[18,19]$

$$
f_{z_{\text {ACO-FBMC }}}(\alpha)=\frac{1}{\sigma \sqrt{2 \pi}} \exp \left(\frac{-\alpha^{2}}{2 \sigma_{1}^{2}}\right) g(\alpha)+\frac{1}{2} \delta(\alpha) .
$$

The PDF of PAM-DMT given by $[18,20]$

$$
f_{z_{\mathrm{PAM}-\mathrm{DMT}}}(\beta)=\frac{1}{\sigma \sqrt{2 \pi}} \exp \left(\frac{-\beta^{2}}{2 \sigma_{2}^{2}}\right) g(\beta)+\frac{1}{2} \delta(\beta) .
$$

The PDF of HACO-OQAM-FBMC can be calculated using convolution of equations (9) and (10).

$$
f_{z_{\text {HACO-FBMC }}}(\gamma)=\int_{-\infty}^{\infty} f_{z_{\text {PAM-DMT }}}(\gamma-\tau) f_{z_{\text {ACO-FBMC }}(t)}(\tau) d \tau .
$$

Substituting equations (9) and (10) in equation (11) gives 


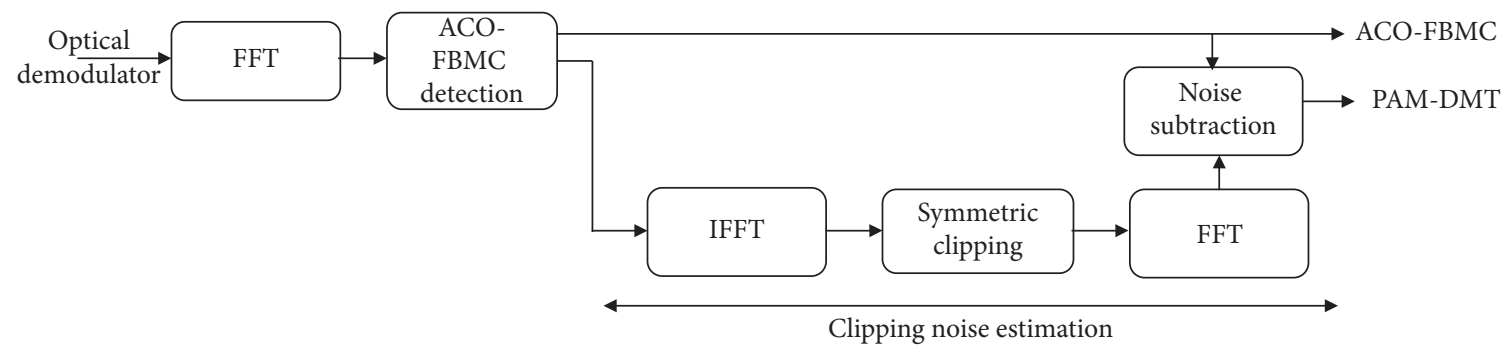

FIgURE 3: Block diagram of ACO-FBMC and PAM-DMT detection.

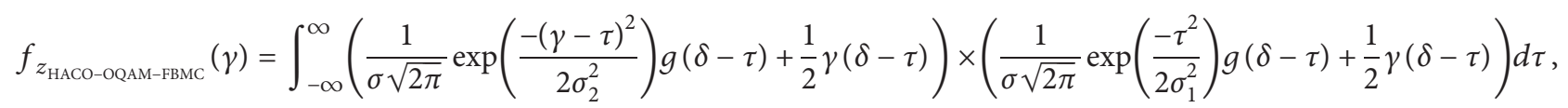

where $\sigma^{2}$ is the variance of the signal and is given by $E\left\{z^{2}\right\}$.

Using identity [21, 22],

$$
\begin{aligned}
& f_{z_{\text {HACO-OQAM-BBMC }}}(\gamma)=\frac{\exp \left(-z^{2} / 2 \sigma_{2}^{2}\right)}{\pi \sigma_{1} \sigma_{2}} \sqrt{\pi\left(\frac{\sigma_{1}^{2} \sigma_{2}^{2}}{2\left(\sigma_{1}^{2}+\sigma_{2}^{2}\right)}\right)} \exp \left(\frac{\gamma^{2}}{\sigma_{2}^{4}}\left(\frac{\sigma_{1}^{2} \sigma_{2}^{2}}{2\left(\sigma_{1}^{2}+\sigma_{2}^{2}\right)}\right)\right) \\
& \cdot\left[Q\left(-\frac{\gamma}{\sigma_{2}^{2}} \sqrt{2\left(\frac{\sigma_{1}^{2} \sigma_{2}^{2}}{2\left(\sigma_{1}^{2}+\sigma_{2}^{2}\right)}\right)}\right)-Q\left(-\frac{\gamma}{\sigma_{2}^{2}} \sqrt{2\left(\frac{\sigma_{1}^{2} \sigma_{2}^{2}}{2\left(\sigma_{1}^{2}+\sigma_{2}^{2}\right)}\right)}+\frac{\gamma}{\sqrt{2\left(\sigma_{1}^{2} \sigma_{2}^{2} / 2\left(\sigma_{1}^{2}+\sigma_{2}^{2}\right)\right)}}\right)\right] \\
& +\frac{1 / 2}{\sqrt{2 \pi}}\left(\frac{1}{\sigma_{1}} \exp \left(\frac{-\gamma^{2}}{2 \sigma_{1}^{2}}\right)+\frac{1}{\sigma_{2}} \exp \left(\frac{-\gamma^{2}}{2 \sigma_{2}^{2}}\right)\right) g(\gamma)+\frac{1}{4} \delta(\gamma) .
\end{aligned}
$$

The optical power of HACO-OQAM-FBMC is given by

$$
P_{\mathrm{opt}}=\int_{0}^{\infty} \gamma f_{z_{\text {HACO-OQAM-PBMC }}}(\gamma) d \gamma .
$$
by

The electrical power of HACO-OQAM-FBMC is given

$$
P_{\mathrm{elc}}=\int_{0}^{\infty} \gamma^{2} f_{z_{\mathrm{HACO}-\mathrm{OQAM}-\mathrm{BBMC}}}(\gamma) d \gamma .
$$

Equations (14) and (15) have no closed-form solution. Therefore, they are calculated using a numerical process.

The probability density function of HACO-OQAMFBMC is given in Figure 4. It consists of both theoretical and simulated PDF and shows similar performance. The PDF curve shows that the proposed technique is power efficient.

3.2. Bit Error Probability (BEP) of HACO-OQAM-FBMC. The bit error probability of HACO-OQAM-FBMC is given by [23]

$$
P_{\sqrt{M}}=2\left(1-\frac{1}{\sqrt{M}}\right) Q\left(\sqrt{\frac{3}{M-1} \frac{S_{\mathrm{av}}}{N_{o}}}\right),
$$

where $M$ is the constellation size and $S_{\mathrm{av}} / N_{o}$ is the average signal to noise ratio (SNR)/symbol.

Figure 5 shows the bit error rate (BER) performance of the HACO-OQAM-FBMC modulation technique. The FBMC overlapping factor $(k=4)$ is used for simulation. The theoretical and simulation results show almost similar performance.

As shown in Figure 6, the performance of HACOOQAM-FBMC shows $2 \mathrm{~dB}$ degradation when compared with ACO-OFDM because only half of the power is allocated to odd subcarriers (ACO) in HACO-OQAM-FBMC. In reality, the performance of the proposed scheme is better when compared with ACO-OFDM because the OFDM performance is degraded due to the use of cyclic prefix (CP) [24]. The BER performance of the proposed technique is better than that of DCO-OFDM and ADO-OFDM because no DC bias is used, and hence, it is power efficient.

Analysis of Figure 7 shows that there is a $3 \mathrm{~dB}$ penalty for HACO-OQAM-FBMC as compared to ACO-FBMC, but actually both have similar performance because half power 


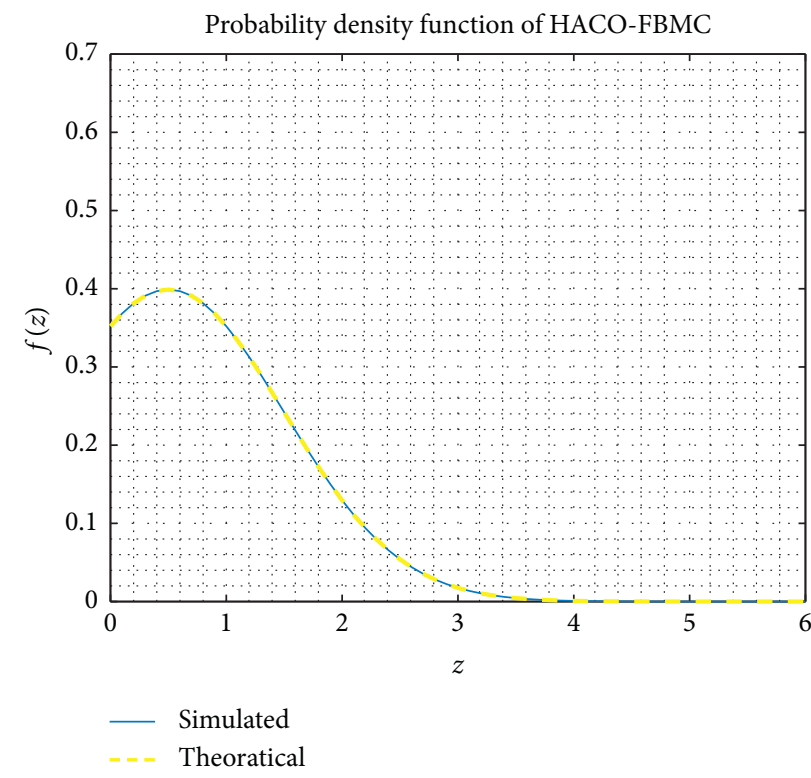

FIgUre 4: Probability density function of HACO-OQAM-FBMC.

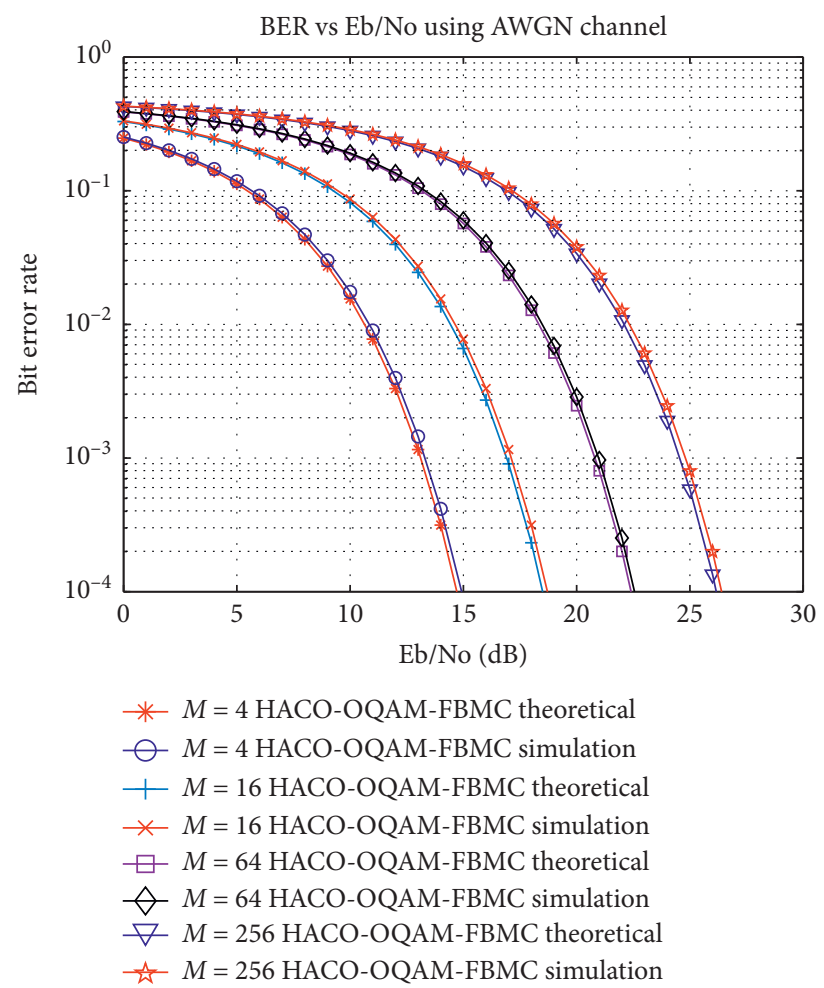

Figure 5: BER of HACO-OQAM-FBMC using 4-OQAM, 16-OQAM, 64-OQAM, and 256-OQAM modulation.

of HACO-OQAM-FBMC is contained by even subcarriers (PAM-DMT). The BER performance of the proposed technique is power efficient as compared to that of DCOFBMC and EHACO-OFDM because no DC bias is used in the proposed technique.

3.3. Peak to Average Power Ratio (PAPR) of HACO-OQAM$F B M C$. The PAPR of HACO-OQAM-FBMC is given by

$$
\operatorname{PAPR}_{\text {HADO }}=10 \log _{10}\left(\frac{\max [z]^{2}}{E\left\{[z]^{2}\right\}}\right) .
$$

The PAPR of ACO-OFDM, ACO-FBMC, DCO-OFDM, EHACO-OFDM, ADO-OFDM, DCO-OFDM, and HACOOQAM-FBMC is given in Figure 8. ACO-OFDM has the best PAPR as compared to other techniques. The proposed method HACO-OQAM-FBMC PAPR can be reduced using 


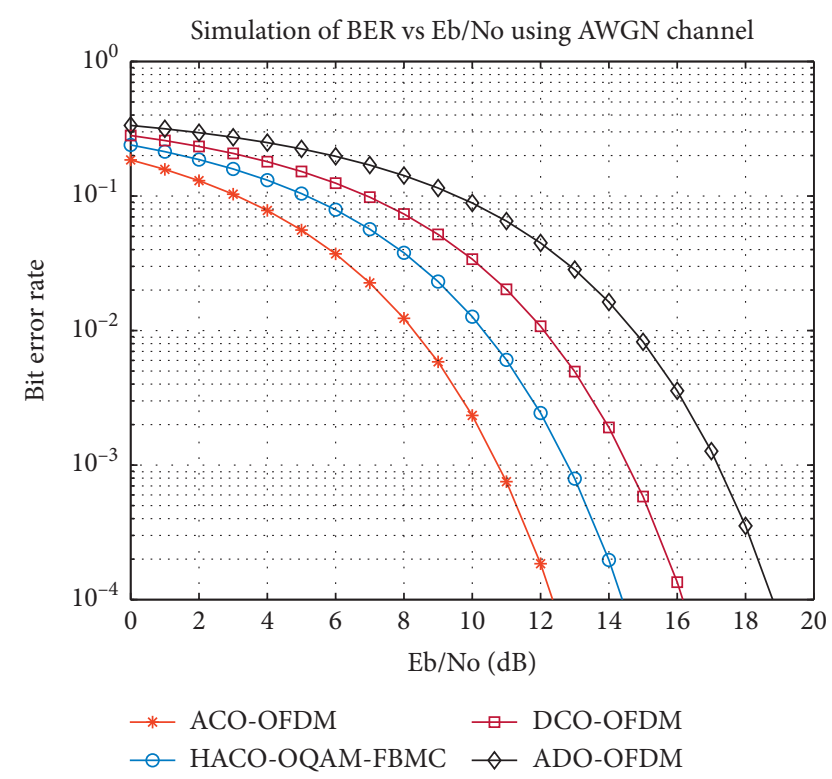

FIGURE 6: Simulation of BER of ACO-OFDM, DCO-OFDM, ADOOFDM, and HACO-OQAM-FBMC.

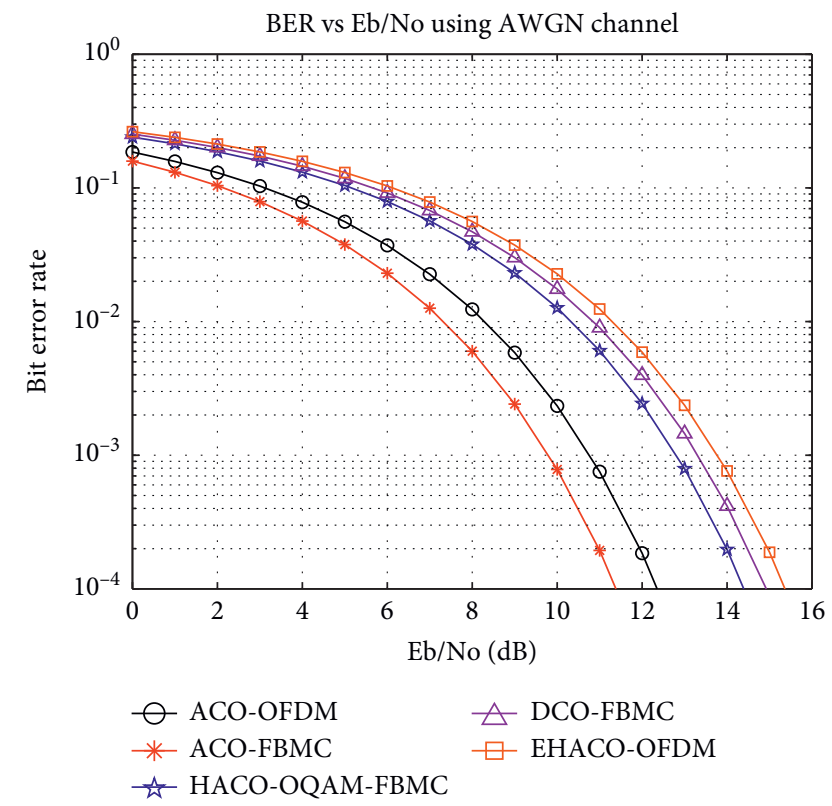

FIgURE 7: Simulation of BER of ACO-FBMC, DCO-FBMC, EHACO-OFDM, and HACO-OQAM-FBMC.

optimization algorithms. DCO-FBMC has the worst PAPR as compared to other techniques because of DC bias and the FBMC modulation technique.

3.4. Computational Complexity of HACO-OQAM-FBMC. The computational complexity of HACO-OQAM-FBMC is given by

$$
\mathrm{RM}_{\mathrm{HF}}=M \times\left(4 \times\left(N \log _{2} N-3 N+4\right)+8 \mathrm{NK}\right),
$$

where $\mathrm{RM}_{\mathrm{HF}}$ is the number of real multiplications for HACO-OQAM-FBMC. $M$ is the constellation size, $N$ is the number of subcarriers, and $K$ is the overlapping factor.

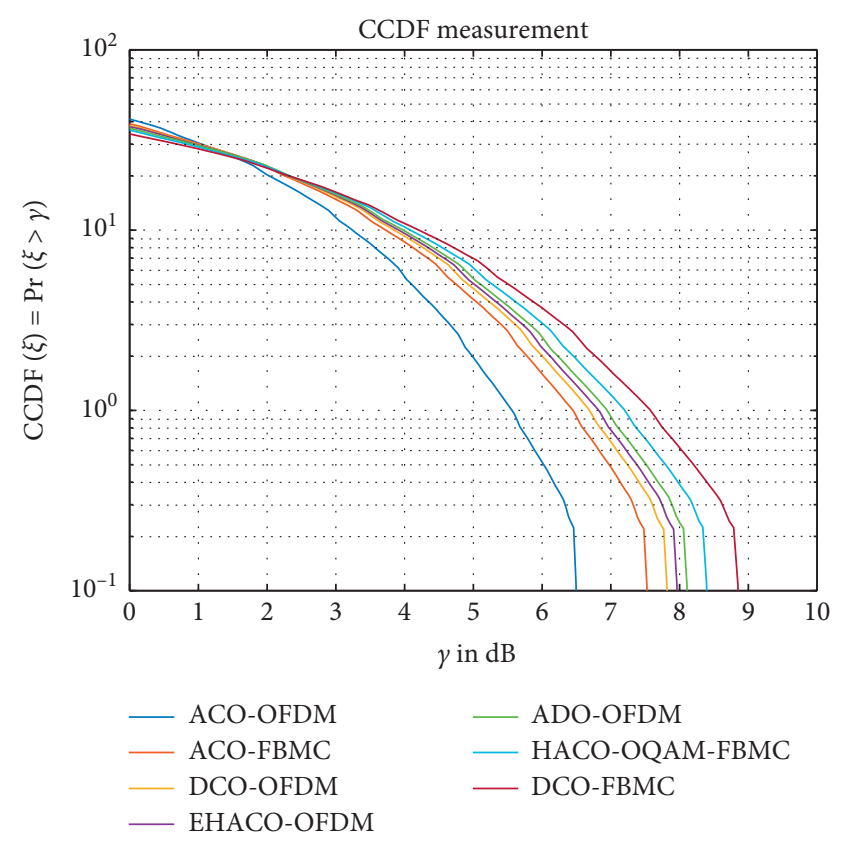

FIGURE 8: Simulation of peak to average power ratio (PAPR) of ACO-OFDM, ACO-FBMC, DCO-OFDM, EHACO-OFDM, ADOOFDM, DCO-OFDM, and HACO-OQAM-FBMC.

$$
\mathrm{RA}_{\mathrm{HF}}=M \times\left(4 \times\left(N \log _{2} N-3 N+4\right)+8 \mathrm{NK}\right),
$$

where $\mathrm{RA}_{\mathrm{HF}}$ is the number of real additions for HACOOQAM-FBMC.

The computational complexity of HACO-OQAMFBMC is given in Figures 9 and 10. The computational complexity of ACO-OFDM is the lowest as compared to other techniques because of the no-DC bias technique and use of OFDM. OFDM has low computational complexity than FBMC [25]. HACO-OQAM-FBMC technique computational complexity is the highest among other techniques because extra processing is required for PAM-DMT signals.

3.5. Spectral Efficiency (SE) of HACO-OQAM-FBMC. The spectral efficiency of HACO-OQAM-FBMC can be calculated from [4]

$$
\eta=\alpha \zeta\left(1-P_{\sqrt{M}}\right) \nu_{T} \nu_{F}
$$

where $\alpha$ is the coding rate of channel, $\zeta=$ Number of bits/Subcarrier $=\log _{2}(M)$, and $\nu_{T} \nu_{F}$ is the time-frequency efficiency.

The spectral efficiency of ACO-OFDM, DCO-OFDM, ACO-FBMC, DCO-FBMC, EHACO-OFDM, and HACOOQAM-FBMC is given in Figure 11. The spectral efficiency of HACO-OQAM-FBMC is higher than that of other modulation techniques and almost similar to that of DCO-FBMC because DCO-FBMC does not use Hermitian symmetry and, hence, has more subcarriers for data communication. All other modulation techniques have low spectral efficiency as compared to the proposed method because they are OFDMbased techniques which have low spectral efficiency as compared to FBMC [25]. 


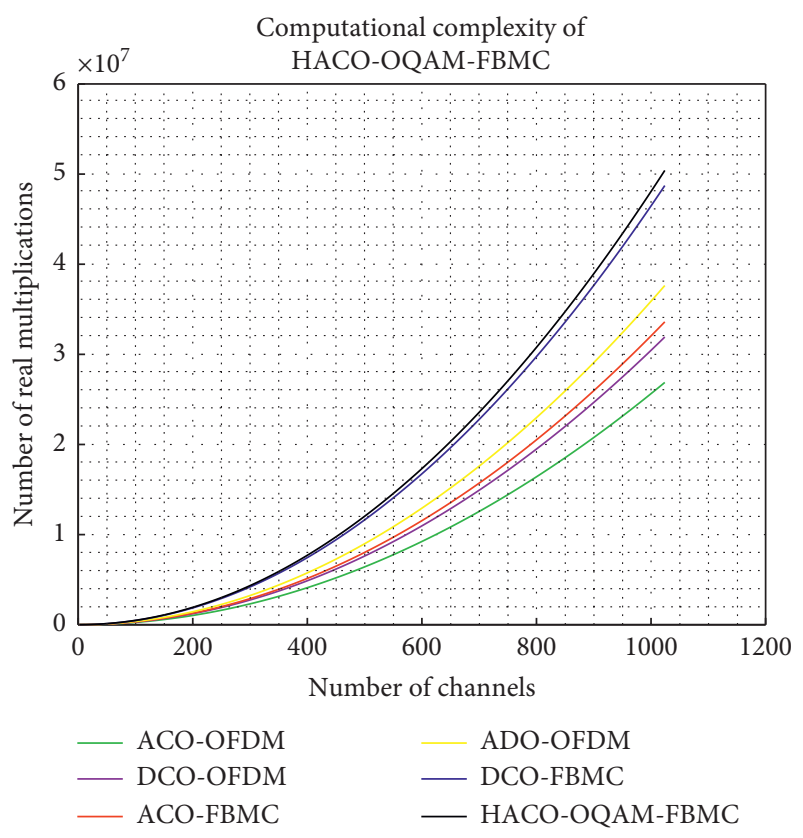

FIGURE 9: Simulation of computational complexity (multiplications) of ACO-OFDM, ACO-FBMC, DCO-OFDM, ADO-OFDM, DCO-OFDM, and HACO-OQAM-FBMC.

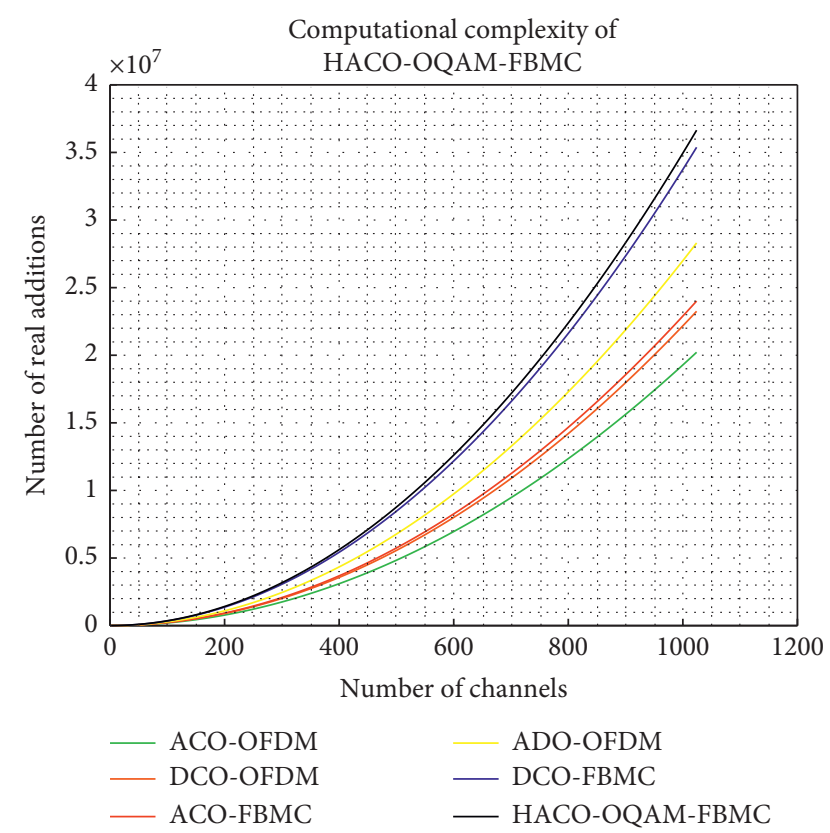

FIGURE 10: Simulation of computational complexity (additions) of ACO-OFDM, ACO-FBMC, DCO-OFDM, ADO-OFDM, DCOOFDM, and HACO-OQAM-FBMC.

3.6. Power Spectral Density (PSD) of HACO-OQAM-FBMC. The power spectral density of HACO-OQAM-FBMC is shown in Figure 12. PSD of ACO-FBMC, DCO-FBMC, and HACO-OQAM-FBMC is the same because the same prototype filter is used. The PSD of the proposed technique is better than that of the OFDM-based technique because FBMC has low out-of-band distortion as compared to OFDM.

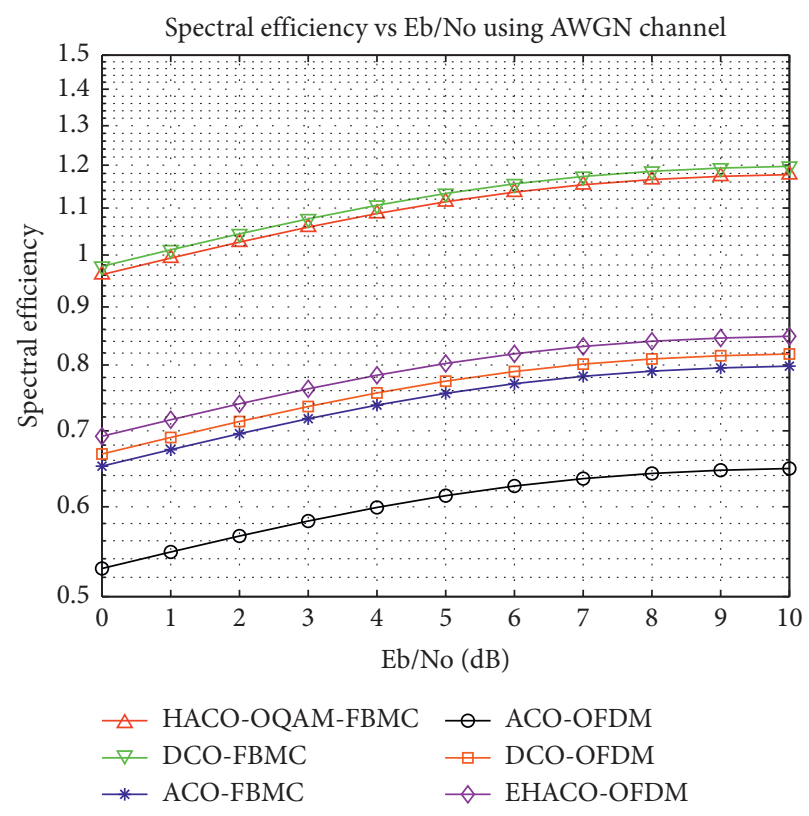

FIgURE 11: Spectral efficiency of HACO-OQAM-FBMC, ACOOFDM, DCO-OFDM, EHACO-OFDM, DCO-FBMC, and ACOFBMC.

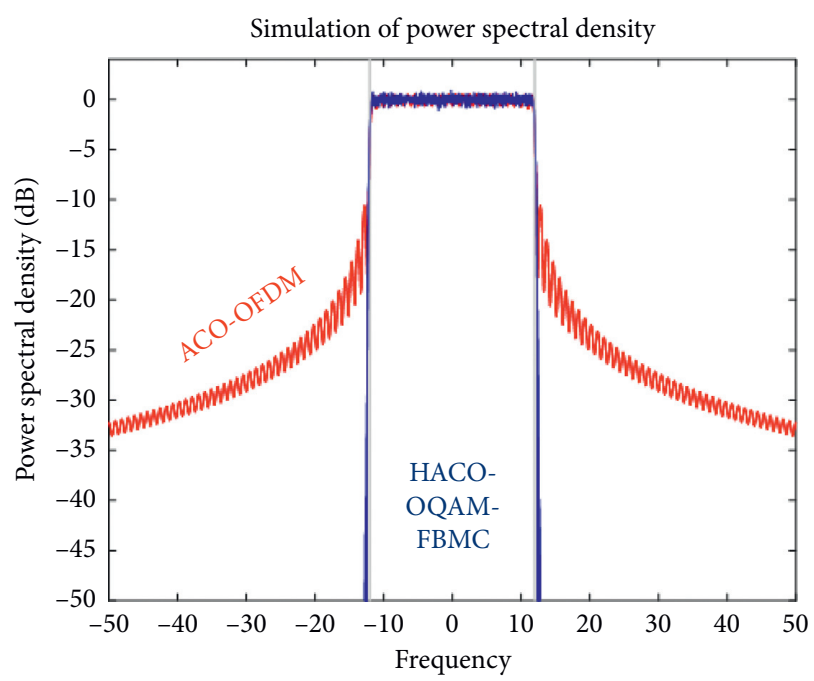

FIGURE 12: Simulation of the power spectral density (PSD) of ACOOFDM and HACO-OQAM-FBMC.

\section{Conclusions}

This research work proposes Hybrid FBMC schemes for visible light communication based on an intensity modulation/direct detection (IM/DD) channel. In hybrid asymmetrically clipped DC biased optical FBMC (HACOOQAM-FBMC), ACO-FBMC is used for the odd subcarriers and for even subcarriers, PAM-DMT is used. The PAPR of the proposed scheme is $0.96 \mathrm{~dB}$ more than ACO-FBMC because of hybridization. The spectral efficiency of HACOOQAM-FBMC is higher than that of ACO-OFDM, DCOOFDM, ACO-FBMC, and EHACO-OFDM due to use of even subcarriers and the FBMC multicarrier modulation 
technique. The computational complexity of HACOOQAM-FBMC is higher because extra processing is required for even subcarriers. It is shown that there is no interference on odd subcarriers because PAM-DMT and receiver demodulation is achieved using the clipping estimation technique. Future directions can be reducing computational complexity and PAPR of HACO-OQAM-FBMC.

\section{Data Availability}

The data used to support the findings of this study in the manuscript are available from the corresponding author upon request.

\section{Conflicts of Interest}

The authors declare that they have no conflicts of interest.

\section{Acknowledgments}

The authors gratefully acknowledge the support of Council of Scientific and Industrial Research (CSIR), New Delhi, under the Senior Research Fellowship grant 09/135/(0798)/ 18-EMR-I.

\section{References}

[1] R. Nissel, S. Schwarz, and M. Rupp, "Filter bank multicarrier modulation schemes for future mobile communications," IEEE Journal on Selected Areas in Communications, vol. 35, no. 8, pp. 1768-1782, 2017.

[2] S. Kumar and P. Singh, "A comprehensive survey of visible light communication: potential and challenges," Wireless Personal Communications, vol. 109, no. 2, pp. 1357-1375, 2019.

[3] X. Zhang, C. Zhang, M. Zhu et al., "SSB pruned DFT-spread FBMC signal with low PAPR in direct-detection PONs," IEEE Photonics Journal, vol. 12, no. 3, 2020.

[4] Y. Liu, X. Chen, Z. Zhong et al., "Waveform design for 5G networks: analysis and comparison," IEEE Access, vol. 5, pp. 19282-19292, 2017.

[5] S. Kumar and P. Singh, "Filter bank multicarrier modulation schemes for visible light communication," Wireless Personal Communications, vol. 113, no. 4, pp. 2709-2722, 2020.

[6] T. C. Bui, S. Kiravittaya, K. Sripimanwat et al., "A comprehensive lighting configuration for efficient indoor visible light communication networks," International Journal of Optics, vol. 14, 2016.

[7] M. A. Al-Jarrah, A. Al-Dweik, K.-H. Park, and M.-S. Alouini, "Amplitude-coherent detection for optical wireless communications: opportunities and limitations," IEEE Open Journal of the Communications Society, vol. 1, pp. 550-562, 2020.

[8] J.-Y. Wang, C. Liu, J.-B. Wang, Y. Wu, M. Lin, and J. Cheng, "Physical-layer security for indoor visible light communications: secrecy capacity analysis," IEEE Transactions on Communications, vol. 66, no. 12, pp. 6423-6436, 2018.

[9] J. Y. Wang, Q. L. Li, J. X. Zhu, and Y. Wang, "Impact of receiver's tilted angle on channel capacity in VLCs," Electronics Letters, vol. 53, no. 6, pp. 421-423, 2017.

[10] J. Armstrong and B. Schmidt, "Comparison of asymmetrically clipped optical OFDM and DC-biased optical OFDM in AWGN," IEEE Communications Letters, vol. 12, no. 5, pp. 343-345, 2008.
[11] X. Zhang, Y. F. Zhou, Y. P. Yu et al., "Comparison and analysis of DCO-OFDM, ACO-OFDM and ADO-OFDM in IM/DD systems," Applied Mechanics and Materials, vol. 701, pp. 1059-1062, 2015.

[12] R. Guan, N. Huang, J. Y. Wang et al., "Enhanced hybrid asymmetrically clipped orthogonal frequency division multiplexing for optical wireless communications," Optical Engineering, vol. 55, no. 5, 2016.

[13] S. Dimitrov, S. Sinanovic, and H. Haas, "Signal shaping and modulation for optical wireless communication," Journal of Lightwave Technology, vol. 30, no. 9, pp. 1319-1328, 2012.

[14] M. G. Bellanger, "Specification and design of a prototype filter for filter bank based multicarrier transmission," IEEE, vol. 4, pp. 2417-2420, 2001.

[15] S. Mirabbasi and K. Martin, "Design of prototype filter for near-perfect-reconstruction overlapped complex-modulated transmultiplexers," IEEE, vol. 1, 2002.

[16] J. Yang and Y. Chen, "Indoor localization using improved rssbased lateration methods," IEEE, vol. 1, 2009.

[17] J. Armstrong and A. J. Lowery, "Power efficient optical OFDM," Electronics Letters, vol. 42, no. 6, pp. 370-372, 2006.

[18] L. Chen, B. Krongold, and J. Evans, "Performance analysis for optical OFDM transmission in short-range IM/DD systems," Journal of Lightwave Technology, vol. 30, no. 7, pp. 974-983, 2012.

[19] D. J. Barros, S. K. Wilson, and J. M. Khan, "Comparison of orthogonal frequency-division multiplexing and pulse-amplitude modulation in indoor optical wireless links," IEEE Transactions on Communications, vol. 60, no. 1, pp. 153-163, 2011.

[20] X. Li, R. Mardling, and J. Armstrong, "Channel capacity of IM/DD optical communication systems and of ACOOFDM," IEEE, vol. 60, pp. 2128-2133, 2007.

[21] I. S. Gradshteyn and I. M. Ryzhik, "Table of integrals, series, and products," 2000.

[22] B. Ranjha and M. Kavehrad, "Hybrid asymmetrically clipped OFDM-based IM/DD optical wireless system," Journal of Optical Communications and Networking, vol. 6, no. 4, pp. 387-396, 2014.

[23] J. G. Proakis and M. Salehi, "Digital communications," 2014.

[24] B. Muquet, Z. Zhengdao Wang, G. B. Giannakis et al., "Cyclic prefixing or zero padding for wireless multicarrier transmissions?," IEEE Transactions on Communications, vol. 50, no. 12, pp. 2136-2148, 2002.

[25] B. de Courville, "OFDM versus filter bank multicarrier," IEEE Signal Processing Magazine, vol. 28, no. 3, pp. 92-112, 2011. 